\title{
EL ANÁLISIS DE LA PLATAFORMA VIRTUAL "REKO” DE LA UNIVERSIDAD TECNOLÓGICA METROPOLITANA DEL ESTADO DE CHILE, DESDE LA MIRADA DE EXPERTOS Y DOCENTES.
}

\section{THE ANALYSIS OF THE VIRTUAL PLATAFORM "REKO" OF THE UNIVERSIDAD TECNOLÓGICA METROPOLITANA DEL ESTADO DE CHILE, FROM THE PERSPECTIVE OF EXPERTS AND PROFESSORS}

\author{
Cristóbal Felipe Moreno Muñoz \\ cristobal.moreno.m@usach.cl \\ Universidad de Santiago de Chile \\ Ximena Alejandra Díaz Orellana \\ Ximenado@hotmail.com \\ Fabián Enrique Jeno Hernández \\ fabian.jeno@usach.cl \\ Universidad Santo Tomás, Chile
}

Recibido: 17 de enero de 2017

Aprobado: 28 de abril de 2017

El análisis de la plataforma virtual "REKO” de la Universidad Tecnológica Metropolitana del

Estado de Chile, desde la mirada de expertos y docentes. Cristóbal Felipe Moreno Muñoz, Ximena Alejandra Díaz Orellana

Fabián Enrique Jeno Hernández

DOI: http://dx.doi.org/10.22458/caes.v8i1.xxxx

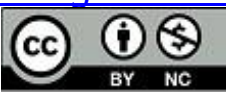

Artículo protegido por licencia Creative Commons 
REVISTA ELECTRÉNICA CALIDAD EN LA EDUCACIÍN SUPERIIRR

Instituta de Gestión de la Calidad Académica

Universidad Estatal a Distancia, Costa Rica
ISSN: 1659-4703, VDL. 8(I) ENERD-JUNIT, 2017: 176 - 206

http://investiga.uned.ac.cг/revistas/index.php/revistacalidad

Carrea: revistacalidad国uned.ac.६Г

DOI: http://dx.doi.org/10.22458/caes.v8i1.xxxx

\section{Resumen}

El siguiente artículo tiene como objetivo analizar la plataforma virtual REKO de la Universidad Tecnológica Metropolitana de Santiago de Chile (UTEM) desde la mirada de diversos actores, bajo un enfoque analítico interpretativo, descriptivo y cualitativo, de aspectos técnicos y pedagógicos. Se emplearon dos instrumentos de recolección de información; en primer lugar, entrevista a dos expertos en el área del diseño y del desarrollo de plataformas virtuales y, en segundo lugar, un grupo focal constituido por ocho docentes de la escuela de diseño de la UTEM. Frente a la información obtenida, mediante estos dos instrumentos, se obtuvieron dos categorías generales de análisis: una que corresponde a los aspectos técnicos y de diseño gráfico de REKO, y otra en relación a los criterios pedagógicos que debiese cumplir dicha plataforma. La investigación propone criterios técnicos y pedagógicos de análisis, que permitirán evaluar dicha plataforma como herramienta de apoyo a la educación superior.

Palabras clave: Plataforma virtual, recursos tecnológicos, tecnología de información y comunicación.

\section{Abstract}

The following article maintains the objective of analyzing the virtual platform REKO, of the Universidad Tecnológica Metropolitana de Santiago de Chile (UTEM), from the perspective of diverse actors under an analytical, interpretative, descriptive and qualitative point of view, applied to technical and pedagogical aspects. Two instruments of recollection were employed: firstly, an interview to two experts in design and virtual platform development and, in second place, a focal group constituted by eight professors of the School of Design of the UTEM. Considering the information obtained, through these two instruments, two general categories of analysis were obtained: one that corresponds to the technical and graphic design aspects of REKO, and another in relation to the pedagogical criteria that said platform must meet. The investigation proposes technical and pedagogical criteria of analysis that will allow the evaluation of this platform as a tool of support in superior education.

Keywords: Virtual Platform, technological resources, informatics and communicational technology.

El análisis de la plataforma virtual “REKO” de la Universidad Tecnológica Metropolitana del

Estado de Chile, desde la mirada de expertos y docentes.

Cristóbal Felipe Moreno Muñoz, Ximena Alejandra Díaz Orellana

Fabián Enrique Jeno Hernández

DOI: http://dx.doi.org/10.22458/caes.v8i1.xxxx

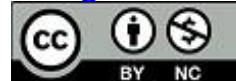

Artículo protegido por licencia Creative Commons 
REVISTA ELECTRÉNICA CALIDAD EN LA EDUCACIÍN SUPERIIRR

Instituta de Gestión de la Calidad Académica

Universidad Estatal a Distancia, Casta Rica
ISSN: 1659-4703, VDL. 8(I) ENERD-JUNIT, 2017: 176 - 206

http://investiga.uned.ac.cr/revistas/index.php/revistacalidad

Carrea: revistacalidad国uned.ac.сг

DOI: http://dx.doi.org/10.22458/caes.v8i1.xxxx

\section{Introducción}

El concepto de tecnologías de la información y la comunicación (TIC's) se refiere al conjunto de tecnologías desarrolladas para gestionar información y enviarla de un lugar a otro y, que contienen un abanico de soluciones muy amplio. Incluyen las tecnologías para almacenar información y recuperarla después; enviar y recibir información de un sitio a otro o procesar información para poder calcular resultados y elaborar informes (Sepúlveda, 2007). Chile es líder regional en el uso y desarrollo de las Tecnologías de Información y Comunicación (TIC's), según el World Economic Forum (2015) y ha incorporado en comunidades estudiantiles, como un apoyo al proceso de enseñanza-aprendizaje, diversos recursos virtuales, por ejemplo: plataformas virtuales, redes virtuales internas (intranet), foros virtuales, redes sociales, etc. Si analizamos esta idea, podemos ver que las TIC's tienen una estrecha relación conceptual con la noción de educación en pos de la entrega de información sin límites ni variables sociales.

Según Altbach (2009), ha habido una desconexión profunda dada por el uso de las nuevas TIC para mejorar la calidad. Ello implica que se están produciendo cambios importantísimos, y este es uno de los elementos primordiales de las transformaciones de la universidad del siglo XXI. Ha cambiado tanto la forma de enseñar como la forma de aprender. Con ello, el rol del docente y el estudiante se debe adaptar a los nuevos objetivos formativos para los alumnos, dado que estos tendrán que formarse para utilizar, usar y producir con los nuevos medios, y el docente tendrá que cambiar sus estrategias de comunicación y asumir su

El análisis de la plataforma virtual “REKO” de la Universidad Tecnológica Metropolitana del

Estado de Chile, desde la mirada de expertos y docentes.

Cristóbal Felipe Moreno Muñoz, Ximena Alejandra Díaz Orellana

Fabián Enrique Jeno Hernández

DOI: http://dx.doi.org/10.22458/caes.v8i1.xxxx

$$
\text { (c) }(\text { i) (5) }
$$


REVISTA ELECTRÍNICA CALIDAD EN LA EDULACIÍN SUPPERIIRR

Instituta de Gestión de la Calidad Académica

Universidad Estatal a Distancia, Costa Rica
ISSN: 1659-4703, VQL. 8(I) ENERD-JUNID, 2017: 176 - 206

http://investiga.uned.ac.cr/revistas/index.php/revistacalidad

Correa: revistacalidad国uned. ac.сГ

DOI: $\underline{\text { http://dx.doi.org/10.22458/caes.v8i1.xxxx }}$

función de facilitador del aprendizaje de los alumnos en entornos cooperativos para ayudarlos a planificar y alcanzar los objetivos.

\section{1.- La educación en pos de la virtualidad}

Actualmente, se habla de los nuevos desafíos que debe considerar la educación y esto se enmarca, principalmente en la adaptación que esta debe asumir en los métodos de enseñanza-aprendizaje en pos de la incorporación de nuevos canales de comunicación y formación de carácter virtual.

Las teorías de aprendizaje tradicionales como el conductismo, el cognitivismo y el constructivismo se han tenido que adecuar a la era digital. En los últimos veinte años, la tecnología ha reorganizado la forma en que vivimos, nos comunicamos y aprendemos. Las necesidades de instrucción y las teorías que describen los principios y procesos de formación, deben reflejar los ambientes sociales subyacentes. En palabras de Vaill: "El aprendizaje debe constituir una forma de ser, un conjunto permanente de actitudes y acciones que los individuos y grupos emplean para tratar de mantenerse al corriente de eventos sorpresivos, novedosos, caóticos, inevitables, recurrentes [...]" (1996).

A partir de todos estos cambios nace el conectivismo, teoría alternativa que presenta un modelo de aprendizaje que reconoce los movimientos tectónicos en una sociedad, en donde el aprendizaje ha dejado de ser una actividad interna e individual. Considerando el aporte de

El análisis de la plataforma virtual "REKO” de la Universidad Tecnológica Metropolitana del

Estado de Chile, desde la mirada de expertos y docentes.

Cristóbal Felipe Moreno Muñoz, Ximena Alejandra Díaz Orellana

Fabián Enrique Jeno Hernández

DOI: http://dx.doi.org/10.22458/caes.v8i1.xxxx

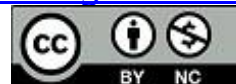

Artículo protegido por licencia Creative Commons 
REVISTA ELEETRÍNICA CALIDAD EN LA EDULACIÍN SULPERIIRR

Instituta de Gestión de la Calidad Académica

Universidad Estatal a Distancia, Casta Rica
ISSN: 1659-4703, VDL. 8(I) ENERD-JUNIT, 2017: 176 - 206

http://investiga.uned.ac.cr/revistas/index.php/revistacalidad

Carrea: revistacalidad国uned.ac.сг

DOI: http://dx.doi.org/10.22458/caes.v8i1.xxxx

esta nueva teoría de aprendizaje y el impacto de la tecnología en los métodos de enseñanza-aprendizaje, los nuevos desafíos que se están implementando actualmente para la educación en pos de la virtualidad, consisten principalmente en el cambio dentro de la relación estudiante-profesor; la búsqueda del trabajo colaborativo y el nexo entre el usuario y el soporte tecnológico.

La Universidad Tecnológica Metropolitana del Estado de Chile (UTEM), como universidad estatal perteneciente al Consejo de Rectores, ha desarrollado una plataforma virtual educativa denominada REKO. Consiste en una Plataforma Virtual de Apoyo a la Academia y tiene por finalidad la administración de asignaturas a través de publicaciones de material educativo digital. Lamentablemente, este sistema no ha logrado un óptimo funcionamiento por diversas razones sociales y tecnológicas. Por ello, se ha escogido esta institución para el desarrollo del perfeccionamiento del sistema de aprendizaje virtual, verificando si este se puede optimizar para suplir las carencias de quienes la imparten y quienes la asimilan.

El problema puntual que pretende abordar esta investigación, consiste en identificar cuáles son las características que tiene la actual plataforma de apoyo a la docencia REKO en las carreras de Diseño Industrial y Diseño en Comunicación Visual, las cuales requieren un conocimiento digital avanzado. Al no hacer uso de estas herramientas, se debe conocer la mirada de los actores, tanto académicos como expertos, que abarcan el uso de este entorno virtual de interacción educativa para revertir esta falencia.

El análisis de la plataforma virtual “REKO” de la Universidad Tecnológica Metropolitana del

Estado de Chile, desde la mirada de expertos y docentes.

Cristóbal Felipe Moreno Muñoz, Ximena Alejandra Díaz Orellana

Fabián Enrique Jeno Hernández

DOI: http://dx.doi.org/10.22458/caes.v8i1.xxxx

$$
\text { (c) }(1)(8)
$$


REVISTA ELECTRÍNICA CALIDAD EN LA EDULACIÍN SUPPERIIRR

Instituta de Gestión de la Calidad Académica

Universidad Estatal a Distancia, Casta Rica
ISSN: 1659-4703, VQL. 8(I) ENERD-JUNIO, 2017: 176 - 206

http://investiga.uned.ac.cr/revistas/index.php/revistacalidad

Carrea: revistacalidad国uned.ac.६Г

DOI: $\underline{\text { http://dx.doi.org/10.22458/caes.v8i1.xxxx }}$

Con este análisis, se busca beneficiar al alumno, al formador docente y a la institución y, para ello, los aspectos que se han escogido analizar corresponden a las características de las plataformas virtuales efectivas: aspectos técnicos-pedagógicos y los aspectos técnicos gráficos.

\section{Criterios pedagógicos de plataformas virtuales}

Producir una plataforma virtual implica el desarrollo de un proyecto que requiere de la adecuada organización y planificación de un conjunto de etapas, fases, actividades y/o tareas que conlleven al desarrollo de escenarios de aprendizaje virtual, guardando los llineamientos y estándares pedagógicos, tecnológicos, organizacionales y estructurales que permitan flexibilidad y movilidad de los contenidos, y actividades de aprendizaje a través de una plataforma web de educación.

Para lograr lo anteriormente mencionado, es necesario contar con un equipo interdisciplinario de profesionales para el diseño y producción de ambientes virtuales de aprendizaje, desarrollar un plan de creación de recursos educativos, lineamientos curriculares para la creación de cursos en línea , un buen un sistema de gestión del aprendizaje y principalmente un modelo (estructura de la asignatura) para el diseño pedagógico, al igual que la producción y el montaje de los cursos.

Se plantea la estructura, modelo y estándar para el diseño de cursos, coherente con las directrices principales, lineamientos institucionales y las orientaciones pedagógicas y

El análisis de la plataforma virtual "REKO” de la Universidad Tecnológica Metropolitana del

Estado de Chile, desde la mirada de expertos y docentes.

Cristóbal Felipe Moreno Muñoz, Ximena Alejandra Díaz Orellana

Fabián Enrique Jeno Hernández

DOI: http://dx.doi.org/10.22458/caes.v8i1.xxxx

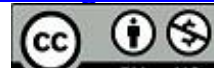

Artículo protegido por licencia Creative Commons 
REVISTA ELEETRÉNICA CALIDAD EN LA EDUCACIÍN SUPERIOR

Instituta de Gestión de la Calidad Académica

Universidad Estatal a Distancia, Costa Rica
ISSN: 1659-4703, VDL. 8(I) ENERD-JUNII, 2017: 176 - 206

http://investiga.uned.ac.cr/revistas/index.php/revistacalidad

Carré: revistacalidad国uned. ac.гг

tecnológicas expuestas para el diseño de ambientes virtuales de aprendizaje. Inicialmente, se describe un análisis de los componentes estructurales de un curso virtual y seguidamente se plantean los estándares necesarios para desarrollar un (lineamiento académico) de alta calidad (Monsiváis, 2014).

Los aspectos de los elementos pedagógicos a considerar se pueden apreciar en detalle en el siguiente esquema:

Criterios pedagógicos para un plataforma virtual

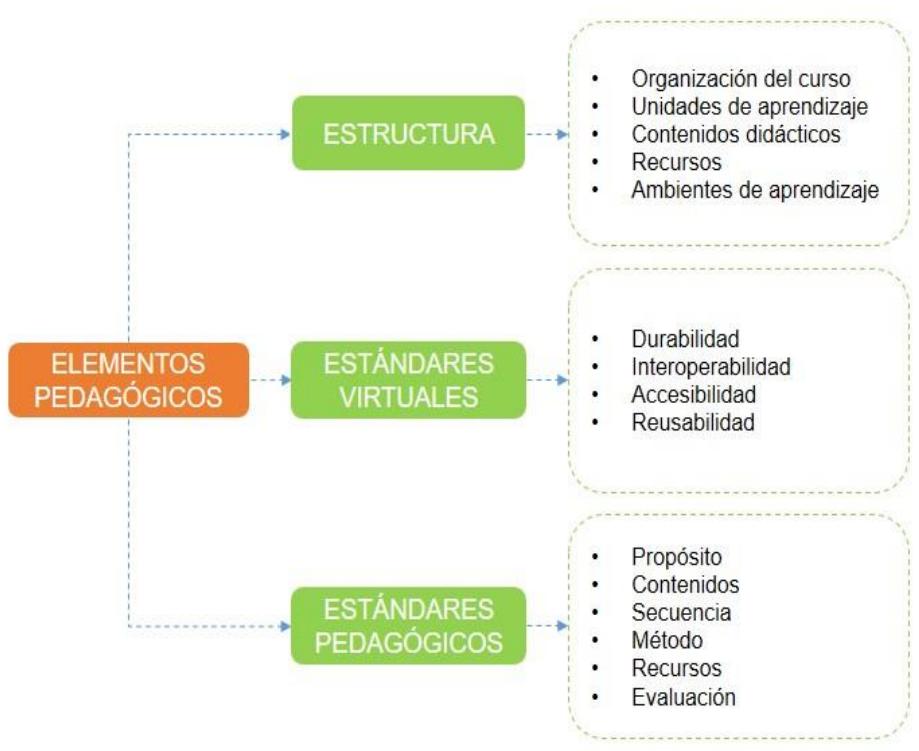

El análisis de la plataforma virtual “REKO” de la Universidad Tecnológica Metropolitana del

Estado de Chile, desde la mirada de expertos y docentes.

Cristóbal Felipe Moreno Muñoz, Ximena Alejandra Díaz Orellana

Fabián Enrique Jeno Hernández

DOI: http://dx.doi.org/10.22458/caes.v8i1.xxxx

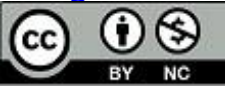

Artículo protegido por licencia Creative Commons 


\section{Criterios técnicos gráficos}

Una buena plataforma virtual no solo debe medirse en aspectos pedagógicos. Es importante tener en consideración los aspectos de diseño, tanto gráficos como visuales y técnicos que conforman el sitio educacional. En aspectos de Diseño Gráfico Comunicacional se clasifican los factores en dimensiones que consideran aspectos gráficos visuales, las características de usabilidad e interfaz y los rasgos técnicos informáticos del sitio web que se pueden apreciar en el siguiente esquema:

El análisis de la plataforma virtual "REKO” de la Universidad Tecnológica Metropolitana del Estado de Chile, desde la mirada de expertos y docentes. Cristóbal Felipe Moreno Muñoz, Ximena Alejandra Díaz Orellana

Fabián Enrique Jeno Hernández

DOI: http://dx.doi.org/10.22458/caes.v8i1.xxxx

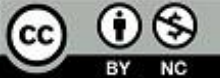

Artículo protegido por licencia Creative Commons 


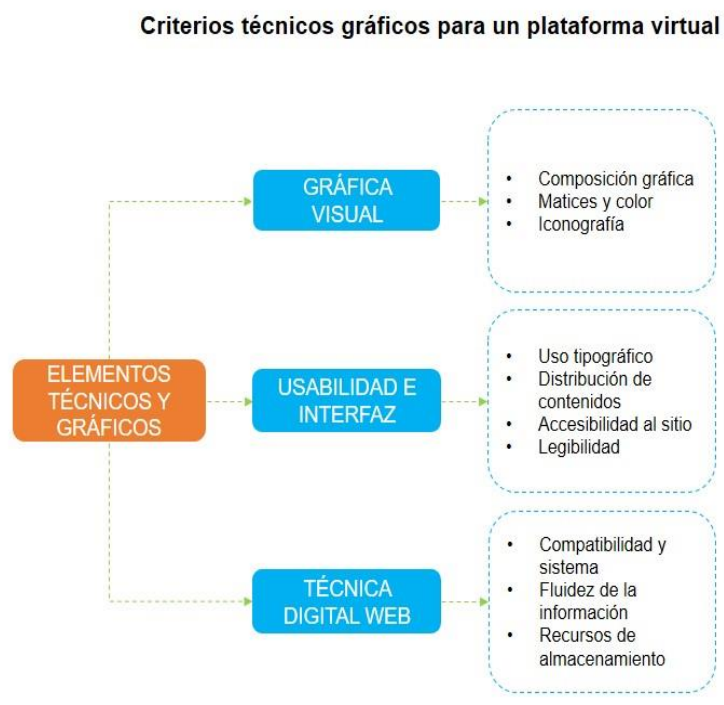

\section{Desarrollo}

La metodología que se empleará en esta investigación corresponde al paradigma analítico interpretativo, que dirige su atención a aquellos aspectos no observables ni susceptibles de cuantificación, tales como creencias, intenciones, motivaciones, interpretaciones, significados. Interesa lo particular y lo contextual, los relatos vividos y, se pretende desarrollar un conocimiento ideográfico que comprende la realidad como dinámica y diversa. También engloba un conjunto de corrientes humanístico-interpretativas, cuyo interés fundamental va dirigido al significado de las acciones humanas y de la vida social. Se busca concebir la educación como proceso social, como experiencia viva para los involucrados en los procesos

El análisis de la plataforma virtual "REKO” de la Universidad Tecnológica Metropolitana del

Estado de Chile, desde la mirada de expertos y docentes.

Cristóbal Felipe Moreno Muñoz, Ximena Alejandra Díaz Orellana

Fabián Enrique Jeno Hernández

DOI: http://dx.doi.org/10.22458/caes.v8i1.xxxx

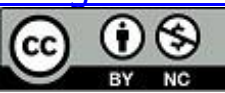

Artículo protegido por licencia Creative Commons 
REVISTA ELEETRÍNICA CALIDAD EN LA EDULACIÍN SULPERIIRR

Instituta de Gestión de la Calidad Académica

Universidad Estatal a Distancia, Casta Rica
ISSN: 1659-4703, VQL. 8(I) ENERD-JUNIO, 2017: 176 - 206

http://investiga.uned.ac.cr/revistas/index.php/revistacalidad

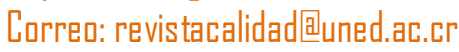

DOI: http://dx.doi.org/10.22458/caes.v8i1.xxxx

y para las instituciones educativas. De esta manera, se enfatiza que, transformando la conciencia de los docentes, estos transformarán su práctica (Miles y Huberman, 1984) y, además, los hechos se interpretarán partiendo de los deseos, intereses y expectativas de los sujetos y no de manera neutral (Ramírez, 2011).

Con ello en mente, el estudio del caso REKO se llevará a cabo en dos etapas, independientes entre sí: primero, a través de entrevistas a expertos y, en segundo lugar, en un grupo focal. La transcripción de ambas etapas se puede encontrar en un anexo de este artículo o directamente en la tesis de la cual formó parte este estudio (Díaz y Moreno, 2014).

Las entrevistas a expertos consistieron en una fase preliminar explicativa, donde cada experto fue orientado sobre los aspectos técnicos a evaluar en la plataforma virtual REKO. Posteriormente, cada entrevistado de forma independiente realizó un análisis y un testeo de la plataforma, ingresando como usuario registrado y como usuario de visita para identificar las falencias de los aspectos técnicos.

Los expertos entrevistados fueron Moisés Andrade Ortiz, Técnico Superior en Gráfica Publicitaria de la Escuela de Arte de Sevilla con estudios superiores de Diseño Gráfico de la Escuela de Arte № 10 de Madrid, y Master en Edición de la Universidad de Salamanca; y Marcos Lorca, Diseñador en Comunicación Virtual de la Universidad Tecnológica Metropolitana, y Magister en Educación con mención en informática Educativa de la Universidad de Chile.

El análisis de la plataforma virtual "REKO” de la Universidad Tecnológica Metropolitana del

Estado de Chile, desde la mirada de expertos y docentes.

Cristóbal Felipe Moreno Muñoz, Ximena Alejandra Díaz Orellana

Fabián Enrique Jeno Hernández

DOI: http://dx.doi.org/10.22458/caes.v8i1.xxxx

$$
\text { (c) }(1)(2)
$$

Artículo protegido por licencia Creative Commons 
REVISTA ELECTRÍNICA CALIDAD EN LA EDULACIÍN SUPPERIIRR

Instituta de Gestión de la Calidad Académica

Universidad Estatal a Distancia, Casta Rica
ISSN: 1659-4703, VDL. 8(I) ENERD-JUNIT, 2017: 176 - 206

http://investiga.uned.ac.cr/revistas/index.php/revistacalidad

Correa: revistacalidad国uned. ac.сГ

DOI: http://dx.doi.org/10.22458/caes.v8i1.xxxx

Específicamente, las temáticas a considerar para la entrevista consistieron en tres temas centrales que a su vez se ramificaron en varios subtemas. Ellos se desglosan de la siguiente manera: gráfica visual, usabilidad e interfaz y técnica digital web y subtemas que se desprenden de ellos. Es importante establecer que, en esta investigación, la gráfica visual se entiende como la composición gráfica del sitio, que implica una buena diagramación del espacio virtual, donde se logren identificar claramente sus contenidos. A su vez, es relevante considerar el uso correcto del color y los matices. En este sentido, es imperativo que tengan tonalidades que no entorpezcan la lectura de los contenidos y no cansen la vista de los usuarios. De esta misma forma, es fundamental que la iconografía permita guiar al usuario hacia las funciones que desea realizar.

Respecto de usabilidad e interfaz, se busca considerar factores como la tipografía que debe ser legible y no interrumpir el desarrollo del trabajo en la plataforma; así como también lo es la distribución de contenidos, la cual no debe saturar de información en un solo espacio.

En cuanto a los aspectos de técnica digital web, se entienden como tal: la compatibilidad de sistema, que hace mencion a si la plataforma es compatible o no con los sistemas web más utilizados tanto como exploradores o sistemas operativos computacionales. Por otro lado, está la fluidez de información, lo que implica verificar si la plataforma es eficaz en el almacenamiento de contenidos y de fácil vinculación con otras plataformas de documentación tales como correos electrónicos, intranet y nubes virtuales.

El análisis de la plataforma virtual "REKO” de la Universidad Tecnológica Metropolitana del

Estado de Chile, desde la mirada de expertos y docentes.

Cristóbal Felipe Moreno Muñoz, Ximena Alejandra Díaz Orellana

Fabián Enrique Jeno Hernández

DOI: http://dx.doi.org/10.22458/caes.v8i1.xxxx

$$
\text { (c) }(1)(8)
$$


REVISTA ELECTRÍNICA CALIDAD EN LA EDULACIÍN SUPPERIIRR

Instituta de Gestión de la Calidad Académica

Universidad Estatal a Distancia, Casta Rica
ISSN: 1659-4703, VDL. 8(I) ENERD-JUNIT, 2017: 176 - 206

http://investiga.uned.ac.cr/revistas/index.php/revistacalidad

Correa: revistacalidad国uned. ac.сГ

DOI: http://dx.doi.org/10.22458/caes.v8i1.xxxx

Los expertos concordaron en los siguientes resultados:

Con respecto al primer subtema: Composición grafica de Gráfica Visual, notaron que la plataforma carece de una jerarquía visual. Se concluyó que cumple su función principal, pero falta mayor dinamismo en su composición general, y simplemente utiliza una plantilla gráfica por defecto de las plataformas virtuales, perdiendo carácter e identidad propia. Esto va de la mano con el siguiente sub tema: Matices y Color, donde se puede establecer que existe una armonía cromática, pero muy básica. Tiende a ser de un tipo más escolar, no refleja principalmente, lo que debería estipular una plataforma virtual universitaria, con una apariencia más seria y profesional. Hay una falta de coherencia con los aspectos cromáticos, con los conceptos institucionales y universitarios. Moisés Andrade opina que, "[l]a [página] ("[...] la página) principal se torna algo escolar, casi infantil. Para mí, los recursos y gamas de colores empleados se alejan de la intención de mostrar un sitio que ofrece contenidos a nivel universitario" (2014, ver anexo). Y, finalmente, en el aspecto de la Iconografía: no son de un carácter propio del diseño de la plataforma. Son genéricos, lo cual puede tener un aspecto tanto positivo como negativo. Positivo, ya que permite ser más reconocible universalmente, pero negativo porque no tiene identidad propia.

Referente al segundo tema Usabilidad e Interfaz, sobre Uso Tipográfico: a pesar de que se utilizan tipografías genéricas y de fácil legibilidad, se genera un leve desorden en la excesiva diversidad y tamaño de estas. Sobre ello, Andrade opina que:

El popurrí tipográfico es algo desconcertante. No hay una jerarquía aparente ni un uso coherente de este elemento. [Por ejemplo,] en el menú lateral [de la plataforma], se usa la tipografía museo, para contenidos arial, y pt-sans para el "Slider" del "Home" la

El análisis de la plataforma virtual "REKO” de la Universidad Tecnológica Metropolitana del

Estado de Chile, desde la mirada de expertos y docentes.

Cristóbal Felipe Moreno Muñoz, Ximena Alejandra Díaz Orellana

Fabián Enrique Jeno Hernández

DOI: http://dx.doi.org/10.22458/caes.v8i1.xxxx

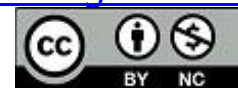

Artículo protegido por licencia Creative Commons 
REVISTA ELEETRÍNICA CALIDAD EN LA EDULACIÍN SUIPERIIRR

Instituta de Gestión de la Calidad Académica

Universidad Estatal a Distancia, Casta Rica
ISSN: 1659-4703, VDL. 8(I) ENERD-JUNIT, 2017: 176 - 206

http://investiga.uned.ac.cr/revistas/index.php/revistacalidad

Carrea: revistacalidad国uned.ac.६Г

DOI: http://dx.doi.org/10.22458/caes.v8i1.xxxx

droid-sans y para barras y [otro] contenidos "Slider" tipografías variadas, manuscritas y de distintas familias tipográficas (Andrade, 2014, ver anexo).

Con respecto al segundo subtema Distribución de contenidos, hay una falta de jerarquía en la información que desorienta al usuario. Esta no permite diferenciar bien las áreas de la plataforma virtual. En el subtema de Accesibilidad al sitio, no hay mayor dificultad a nivel usuario, pero sí carece de conectividad con sistemas más avanzados, como aquellos usados en organismos gubernamentales. Lorca (2014, ver anexo) aclara que "[...]no pasa validación en W3C del código HTML y CCS (lenguajes informáticos de desarrollo de plataformas web), el cual es un requerimiento para organismos estatales". $\mathrm{Y}$ finalmente, en el subtema Legibilidad, se encontró que la misma carencia de una estructura jerárquica de la información provoca que ciertos aspectos sean poco legibles y por ende poco intuitivos para el usuario.

En el tercer tema Técnica Digital Web, los entrevistados no mostraron problemas con respecto a la Compatibilidad y Sistema. Con respecto a la Fluidez de la información, la plataforma operó bien, al igual que Recursos y almacenamiento. Andrade hace hincapié en que, "El uso de gestores de contenidos con base de datos con fuentes de información y almacenamiento es una buena elección para trabajar con grandes fluidos de información y usuarios registrados (2014, ver anexo). Esto da a entender que dentro de esta entrevista este punto es el más favorable y es el que menor análisis crítico requiere.

El análisis de la plataforma virtual “REKO” de la Universidad Tecnológica Metropolitana del

Estado de Chile, desde la mirada de expertos y docentes.

Cristóbal Felipe Moreno Muñoz, Ximena Alejandra Díaz Orellana

Fabián Enrique Jeno Hernández

DOI: http://dx.doi.org/10.22458/caes.v8i1.xxxx

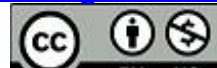

Artículo protegido por licencia Creative Commons 
REVISTA ELECTRÍNICA CALIDAD EN LA EDULACIÍN SUPPERIIRR

Instituta de Gestión de la Calidad Académica

Universidad Estatal a Distancia, Casta Rica
ISSN: 1659-4703, VDL. 8(I) ENERD-JUNIT, 2017: 176 - 206

http://investiga.uned.ac.cr/revistas/index.php/revistacalidad

Carrea: revistacalidad国uned.ac.сг

DOI: http://dx.doi.org/10.22458/caes.v8i1.xxxx

Por otra parte, el grupo focal fue formado por ocho docentes, siete hombres y una mujer, entre las edades de 44 y 58 años, de las carreras de Diseño Industrial y/o Diseño en Comunicación visual de la UTEM. Los participantes discutieron y dieron su opinión referente al uso de la plataforma a partir de su propia experiencia como usuario docente.

En este contexto, cabe recordar que esta instancia fue moderada por Ximena Díaz y Cristóbal Moreno (2014), quienes se encargaron de seguir el guion dado, enfocar el diálogo hacia la temática central, garantizar la calidad de la grabación y mantener vivo el interés de los participantes para sacar el máximo provecho de las intervenciones de estos. Al final del grupo focal, las respuestas fueron recogidas y analizadas objetivamente según los parámetros dados por Bertoldi que establece que,

se lleva a cabo un estudio de los protocolos con la transcripción con la finalidad de identificar la presencia de ideas, sus variantes, los detalles que suscitan polémica, las opiniones recurrentes; y con la interpretación, el moderador descubre cuál es la lógica y los esquemas perceptuales que dan sentido a las ideas y opiniones de los participantes (Bertoldi, 2006) poner numero de pagina.

El grupo focal se realizó el 29 de abril de 2014 y tuvo una duración de 90 minutos. Se llevó a cabo en la Sala de Consejos en la Escuela de Diseño de la Facultad de Ciencias de la Tecnología y de la Comunicación Social en la UTEM. En ella se registró, tanto audio como imagen y se debatió la siguiente temática: "Evaluación pedagógica del actual Entorno Virtual de Interacción Educativa REKO de la Universidad Tecnológica Metropolitana en las carreras de Diseño en Comunicación Visual y Diseño Industrial". Su objetivo fue evaluar elementos

El análisis de la plataforma virtual “REKO” de la Universidad Tecnológica Metropolitana del

Estado de Chile, desde la mirada de expertos y docentes.

Cristóbal Felipe Moreno Muñoz, Ximena Alejandra Díaz Orellana

Fabián Enrique Jeno Hernández

DOI: http://dx.doi.org/10.22458/caes.v8i1.xxxx

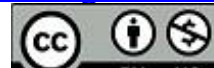

Artículo protegido por licencia Creative Commons 
REVISTA ELEETRÍNICA CALIDAD EN LA EDULACIÍN SUIPERIIRR

Instituta de Gestión de la Calidad Académica

Universidad Estatal a Distancia, Casta Rica
ISSN: 1659-4703, VQL. 8(I) ENERD-JUNI0, 2017: 176 - 206

http://investiga.uned.ac.cr/revistas/index.php/revistacalidad

Carrea: revistacalidad国uned. ac.СГ

DOI: http://dx.doi.org/10.22458/caes.v8i1.xxxx

pedagógicos de dicha plataforma desde la mirada de académicos que deben utilizar este tipo de apoyo en la docencia.

En la transcripción se descubrió que surgieron los siguientes temas y subtemas: accesibilidad, efectividad, comparación con recursos tradicionales (presenciales), utilidad como instrumento de evaluación, dependencia de la respuesta interés de los alumnos, compatibilidad con las experiencias prácticas, actualización como recurso tecnológico según lo que ofrece el mercado. Estos contenidos se trataron a través de preguntas abiertas que permitiesen un diálogo entre los docentes para dar paso a opiniones basadas en experiencias personales.

Sobre accesibilidad se comentó que esta es poco abordable, es decir, que el ingreso a la plataforma no es fácil ni inmediato: "Normalmente, la estoy usando para mensajería solamente, ya que la plataforma no es demasiado amistosa" (Entrevistado 3). Cabe recordar que REKO se presenta como un recurso de acceso acotado por parte de los docentes, debido principalmente a que no es de fácil uso, los recursos de que disponen no se presentan amigables en su uso. Según Sebastián Díaz (2009), toda plataforma virtual debe de tener requisitos mínimos para que se cumpla el proceso de enseñanza - aprendizaje. Entre estos, se encuentran herramientas de gestión de contenidos, de comunicación y colaboración, seguimiento, administración y, por último, complementarias.

El análisis de la plataforma virtual "REKO" de la Universidad Tecnológica Metropolitana del

Estado de Chile, desde la mirada de expertos y docentes.

Cristóbal Felipe Moreno Muñoz, Ximena Alejandra Díaz Orellana

Fabián Enrique Jeno Hernández

DOI: http://dx.doi.org/10.22458/caes.v8i1.xxxx

(c) (i) $(5)$

Artículo protegido por licencia Creative Commons 
REVISTA ELECTRÍNICA CALIDAD EN LA EDULACIÍN SUPPERIIRR

Instituta de Gestión de la Calidad Académica

Universidad Estatal a Distancia, Casta Rica
ISSN: 1659-4703, VDL. 8(I) ENERD-JUNIT, 2017: 176 - 206

http://investiga.uned.ac.cr/revistas/index.php/revistacalidad

Correa: revistacalidad国uned. ac.сГ

DOI: http://dx.doi.org/10.22458/caes.v8i1.xxxx

Además de tener en cuenta estas recomendaciones, la plataforma que decidamos escoger debe adaptarse a nosotros, a nuestros conocimientos y a nuestras maneras de trabajar. Otro aspecto que se considera dentro de la Accesibilidad está asociado a la elección de otros medios y redes, más fáciles de usar, por parte de los estudiantes o profesores. Sobre ellos se comentó que "[...] los alumnos crean cursos por Facebook y en otras plataformas que son más informales, pero sirven para comunicarse" (Entrevistado 4).

En cuanto a Efectividad, se determinó que esta no cumple con esta categoría y ello disminuye su uso. Los entrevistados destacan que: "Muchos no descargan los documentos y la mayoría [de los alumnos] no se entera de los avisos. Solo lo hacen por los correos paralelos. Nunca me ha dado mucho resultado" (Entrevistado 7).

Para los docentes es imprescindible poder enviar archivos de diferentes formatos a los alumnos. La plataforma cumple con estos requisitos, mas no es comparable con plataformas como redes sociales donde la fluidez y la carga y descarga de archivos es mucho más intuitiva. Esta falencia podría solucionarse mediante una mayor dedicación y preparación por parte de los docentes, ya que hoy en día ellos necesitan estar preparados para ofrecer a sus estudiantes oportunidades de aprendizaje apoyadas en las TIC.

Cabe recordar que los estudiantes universitarios actuales (18 y 24 años) son hablantes nativos del lenguaje digital de las computadoras, los video juegos e internet. Por más tecnófilos que los docentes pretendan ser, los individuos entre 35 y 55 años no son nativos

El análisis de la plataforma virtual “REKO” de la Universidad Tecnológica Metropolitana del

Estado de Chile, desde la mirada de expertos y docentes.

Cristóbal Felipe Moreno Muñoz, Ximena Alejandra Díaz Orellana

Fabián Enrique Jeno Hernández

DOI: http://dx.doi.org/10.22458/caes.v8i1.xxxx

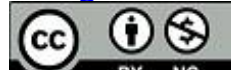

Artículo protegido por licencia Creative Commons 
REVISTA ELECTRÍNICA CALIDAD EN LA EDULACIÍN SUPPERIIRR

Instituta de Gestión de la Calidad Académica

Universidad Estatal a Distancia, Costa Rica
ISSN: 1659-4703, VDL. 8(I) ENERD-JUNIT, 2017: 176 - 206

http://investiga.uned.ac.cr/revistas/index.php/revistacalidad

Carrea: revistacalidad国uned.ac.сг

DOI: http://dx.doi.org/10.22458/caes.v8i1.xxxx

digitales. Al contrario, son inmigrantes digitales y difícilmente sobrepasarán esta categoría, por lo cual la labor del docente que implica recursos digitales proporcionados, por ejemplo, por una plataforma virtual, debe asumir este rol de mediador tecnológico intergeneracional.

Respecto de su Comparación con otros recursos tradicionales como la asistencia presencial, se determinó que no existe comparación. Se destaca que, "No podemos olvidar que, en asignaturas prácticas, existe un aprendizaje por imitación, es decir, participan al menos dos personas, en donde el alumno, al observar, logra su aprendizaje" (Entrevistado 6). Ello tiene relación directa con el modelo tradicional de la enseñanza, llamado aprendizaje social, el cual se basa en una situación social en la que, al menos, participan dos personas: el modelo que realiza una acción determinada, y el sujeto, que realiza la observación de esta, y cuya observación determina la obtención de conocimiento.. A diferencia del aprendizaje por condicionamiento, en el social el que se ilustra no recibe el refuerzo, sino que éste recae, en todo caso, en el modelo. Aquí el que aprende lo hace imitando la conducta del modelo. Además, los docentes encuentran que las clases presenciales son irremplazables por las dinámicas que estas permiten, tales como la socialización, el refuerzo inmediato, el conocimiento progresivo y la retroalimentación.

Sobre la categoría del Proceso de enseñanza-aprendizaje, se determinó que no es óptima. Uno de los entrevistados rescata que,

[...]desde el punto de la evaluación, (el proceso de enseñanza-aprendizaje), depende de las asignaturas. Aquí hay cinco o seis (docentes] que somos de asignaturas teórico-prácticas y, en ese sentido, la parte práctica es muy difícil de evaluar desde

El análisis de la plataforma virtual "REKO” de la Universidad Tecnológica Metropolitana del

Estado de Chile, desde la mirada de expertos y docentes.

Cristóbal Felipe Moreno Muñoz, Ximena Alejandra Díaz Orellana

Fabián Enrique Jeno Hernández

DOI: http://dx.doi.org/10.22458/caes.v8i1.xxxx

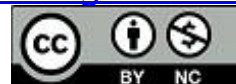

Artículo protegido por licencia Creative Commons 
REVISTA ELEETRÍNICA CALIDAD EN LA EDULACIÍN SULPERIIRR

Instituta de Gestión de la Calidad Académica

Universidad Estatal a Distancia, Costa Rica
ISSN: 1659-4703, VQL. 8(I) ENERD-JUNID, 2017: 176 - 206

http://investiga.uned.ac.cr/revistas/index.php/revistacalidad

Carrea: revistacalidad国uned.ac.६Г

DOI: http://dx.doi.org/10.22458/caes.v8i1.xxxx

una plataforma. Pero sí sería posible en otras [asignaturas], como seminarios, donde podrían utilizarlas (Entrevistado 3).

Este proceso de enseñanza-aprendizaje se está evaluando según un conjunto de estrategias, métodos y tendencias concretas que se utilizan cuando se quiere aprender algo. De ahí surge la clasificación de canales de educación (visual, auditivo y kinestésico). La información seleccionada se organiza y relaciona en el modelo de hemisferio cerebral dominante, y una vez organizada la información, se utiliza de diferente manera: activa, teórica, reflexiva, pragmática, que son los estilos de aprendizaje. Es importante destacar que, según Gardner, el proceso de evaluación también va directamente relacionado con el estilo de enseñanza, que puede ser presencial o a distancia (2005).

En una evaluación presencial, la enseñanza se da de manera directa, mediante instrumentos, situaciones, recursos y procedimientos para obtener información sobre el proceso de aprendizaje, mediante: la observación, el registro del grado de presencia de atributos, el desempeño de los contenidos y procedimientos (Díaz 2010). Por otra parte, la evaluación a distancia necesita de una formalización y planificación anticipada de los contenidos a impartir, además de ser indispensable espacios especializados para proporcionar los recursos necesarios y suficientes, tomando en cuenta que la comunicación y retroalimentación con el estudiante debe ser constante.

El análisis de la plataforma virtual “REKO” de la Universidad Tecnológica Metropolitana del

Estado de Chile, desde la mirada de expertos y docentes.

Cristóbal Felipe Moreno Muñoz, Ximena Alejandra Díaz Orellana

Fabián Enrique Jeno Hernández

DOI: http://dx.doi.org/10.22458/caes.v8i1.xxxx

\section{(c) (i) (5)}


REVISTA ELECTRÍNICA CALIDAD EN LA EDULACIÍN SUPPERIIRR

Instituta de Gestión de la Calidad Académica

Universidad Estatal a Distancia, Casta Rica
ISSN: 1659-4703, VDL. 8(I) ENERD-JUNIT, 2017: 176 - 206

http://investiga.uned.ac.cr/revistas/index.php/revistacalidad

Correa: revistacalidad国uned. ac.сГ

DOI: http://dx.doi.org/10.22458/caes.v8i1.xxxx

Sobre la categoría del Interés del alumno, se considera que la efectividad de la plataforma depende en gran medida de cuánto interés demuestra el estudiante por aprender. Se rescata que:

[...] La sensación que yo tengo, al hacer clases a alumnos de primero y cuarto es que el de cuarto año se hace cargo de más cosas y el de primer año puede aprender lo que quiera siempre que quiera hacerlo, si hay una motivación él va a aprender y le va a interesar y si el lenguaje y la dinámica del curso es la correcta, tanto presencial como virtual él va a embarcarse en el viaje en diferentes niveles (Entrevista 4).

Según Vermunt, los estudiantes son procesadores activos de la información, no son meros receptores pasivos. Un estudiante construye, modifica y utiliza su conocimiento para interpretar situaciones en un determinado dominio y actuar dentro de él (1999). De esta manera, se entiende que es un individuo generalmente maduro con una historia vivencial llena de experiencia, conocimientos, capacidades, hábitos, actitudes, conductas e interés en participar en su propio proceso de formación; características que, condicionan, filtran y, previsiblemente, mejoran los futuros aprendizajes de manera autónoma e independiente, y es precisamente esta autonomía, la que depende de la disposición del alumno a aprender. De esta manera, el docente requiere un proceso tecnológico más depurado, sobre todo de la planificación previa, que las instituciones educativas de carácter presencial (García 2009).

Sobre la sexta categoría respecto de la Experiencia práctica, se considera que es de carácter irremplazable y es imposible obtenerla a partir de un recurso exclusivamente tecnológico. El grupo focal comenta que:

El análisis de la plataforma virtual “REKO” de la Universidad Tecnológica Metropolitana del

Estado de Chile, desde la mirada de expertos y docentes.

Cristóbal Felipe Moreno Muñoz, Ximena Alejandra Díaz Orellana

Fabián Enrique Jeno Hernández

DOI: http://dx.doi.org/10.22458/caes.v8i1.xxxx

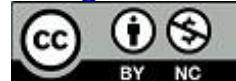

Artículo protegido por licencia Creative Commons 
REVISTA ELECTRÍNICA CALIDAD EN LA EDULACIÍN SUPPERIIRR

Instituta de Gestión de la Calidad Académica

Universidad Estatal a Distancia, Casta Rica
ISSN: 1659-4703, VDL. 8(I) ENERD-JUNID, 2017: 176 - 206

http://investiga.uned.ac.cr/revistas/index.php/revistacalidad

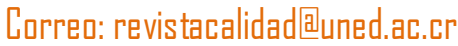

DOI: http://dx.doi.org/10.22458/caes.v8i1.xxxx

La plataforma no puede reemplazar ciertas relaciones con la realidad, primero porque la experiencia con el entorno es de plena libertad para el ser humano. Puedo tener acceso a través de las plataformas, pero cuando voy al terreno y conozco a los [alumnos] antes de trabajar [con] ellos, eso no es reemplazable por lo digital y eso es importante. Puede haber mucha simulación pero hay cosas que no pueden simularse y la experiencia es insustituible. La plataforma está bien en lo que [es] la distribución de contenido y organización, pero en la experiencia con la realidad es insustituible (Entrevistado 5).

Aunque los contenidos teóricos son necesarios para avanzar en el aprendizaje de cualquier área de conocimiento, para poder lograr retenerlos y dotarlos de utilidad es imperativo experimentar con ellos. En la actualidad, bajo la denominación "aprender haciendo" ha surgido una metodología pedagógica que aboga por implantar este tipo de instrucción experimental en los sistemas educativos. Sobre todo, en los más centrados en la adquisición de conocimientos y la evaluación de los mismos, que en la valoración de las aptitudes prácticas que adquieren los estudiantes con ellos.

Para llevar a la práctica una actividad pedagógica basada en la acción, Schank (2013) propone la aplicación de nuevas metodologías didácticas en las aulas que entreguen a los estudiantes aprendizajes experimentales.

Aun así, hay recursos tecnológicos, que la literatura señala, que posibilitan o facilitan el aprendizaje. Sabemos que estos recursos inciden en nuestras vidas y lo hacen también en la forma en que aprendemos, pero será necesario también controlar sus objetivos para lograr una educación democratizadora en la que circule la información.

El análisis de la plataforma virtual “REKO” de la Universidad Tecnológica Metropolitana del

Estado de Chile, desde la mirada de expertos y docentes.

Cristóbal Felipe Moreno Muñoz, Ximena Alejandra Díaz Orellana

Fabián Enrique Jeno Hernández

DOI: http://dx.doi.org/10.22458/caes.v8i1.xxxx

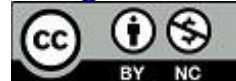

Artículo protegido por licencia Creative Commons 
REVISTA ELECTRÍNICA CALIDAD EN LA EDULACIÍN SUPPERIIRR

Instituta de Gestión de la Calidad Académica

Universidad Estatal a Distancia, Casta Rica
ISSN: 1659-4703, VQL. 8(I) ENERD-JUNI0, 2017: 176 - 206

http://investiga.uned.ac.cr/revistas/index.php/revistacalidad

Carrea: revistacalidad国uned. ac.СГ

DOI: http://dx.doi.org/10.22458/caes.v8i1.xxxx

Se debe cambiar la lógica del tiempo y la estructuración del saber, dejando de lado la linealidad del conocimiento, por la utilización de redes conceptuales, y permitir que los saberes vayan dándose sin la atadura de tiempo, sino como conocimientos múltiples, impredecibles y diversos. Es necesario mejorar la organización; flexibilizar y dar rapidez al acceso a la información en un ambiente cómodo para el alumno, en donde el aprendizaje no esté sujeto a una sola fuente, sino a todas las existentes que aporten a la adquisición de destrezas y pensamientos abiertos.

Para ello, es necesario redefinir constantemente el rol de las tutorías y ayudas al alumno para facilitar su proceso de aprendizaje, su autoevaluación y metacognición, así como también su relación con pares y con recursos (correos electrónicos, foros y listas de discusión, biblioteca virtual, entre otros).

La séptima y última categoría es sobre el Estado de la plataforma respecto a otros recursos tecnológicos que disponen y utilizan los alumnos. El grupo focal considera que está desactualizada y se destaca que la plataforma REKO resulta ajena y poco funcional como un sistema abierto. Como proceso, "funciona solo para algunas cosas y ahí, yo veo una dificultad" (Entrevistado 2).

Para esta conversación, cabe recordar que la web ha evolucionado desde su creación y sus actualizaciones han dado paso a una variedad de herramientas. Entre ellas, se destaca la

El análisis de la plataforma virtual “REKO” de la Universidad Tecnológica Metropolitana del

Estado de Chile, desde la mirada de expertos y docentes.

Cristóbal Felipe Moreno Muñoz, Ximena Alejandra Díaz Orellana

Fabián Enrique Jeno Hernández

DOI: http://dx.doi.org/10.22458/caes.v8i1.xxxx

(c) (i) (5)

Artículo protegido por licencia Creative Commons 
REVISTA ELECTRÍNICA CALIDAD EN LA EDULACIÍN SUPPERIIRR

Instituta de Gestión de la Calidad Académica

Universidad Estatal a Distancia, Costa Rica
ISSN: 1659-4703, VQL. 8(I) ENERD-JUNID, 2017: 176 - 206

http://investiga.uned.ac.cr/revistas/index.php/revistacalidad

Carrea: revistacalidad国uned.ac.६Г

DOI: http://dx.doi.org/10.22458/caes.v8i1.xxxx

posibilidad de establecer redes sociales que conforman comunidades, en donde los usuarios puedan incluir tanto opiniones como archivos audiovisuales, compartir y descargar diferentes tipos de recursos y así, facilitar la participación y colaboración.

A modo corolario, es posible observar a través de los relatos, que los docentes participantes del grupo focal, mayoritariamente evalúan negativamente el uso de la plataforma como apoyo para el proceso de enseñanza aprendizaje, principalmente, porque es poco accesible, solo actúa como mensajería, no es posible usarla como herramienta de evaluación del proceso de enseñanza-aprendizaje y no se usa porque hay preferencia por lo presencial, entre otros factores.

En respuesta a lo anterior, se debe tener presente que los medios son meros vehículos que proporcionan instrucción. Los atributos de un medio son sus capacidades, siempre presentes con el fin de ser usadas para influir en el aprendizaje de los estudiantes. En este modelo pedagógico se plantea cómo el centro, el alumno es objetivo de todos los procesos de aprendizaje y sujeto de la acción formativa, ya que es quien aprende y a quien se enseña. Interactúa con su tutor por medio de las herramientas tecnológicas y además participa grupalmente con otros compañeros también.

\section{Conclusiones}

El análisis de la plataforma virtual "REKO” de la Universidad Tecnológica Metropolitana del

Estado de Chile, desde la mirada de expertos y docentes.

Cristóbal Felipe Moreno Muñoz, Ximena Alejandra Díaz Orellana

Fabián Enrique Jeno Hernández

DOI: http://dx.doi.org/10.22458/caes.v8i1.xxxx

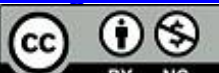

Artículo protegido por licencia Creative Commons 
REVISTA ELEETRÍNICA CALIDAD EN LA EDULACIÍN SUPPERIIRR

Instituta de Gestión de la Calidad Académica

Universidad Estatal a Distancia, Casta Rica
ISSN: 1659-4703, VDL. 8(I) ENERD-JUNIT, 2017: 176 - 206

http://investiga.uned.ac.cr/revistas/index.php/revistacalidad

Correa: revistacalidad国uned. ac.сГ

DOI: http://dx.doi.org/10.22458/caes.v8i1.xxxx

Esta investigación consistió en identificar, cuáles son las características que tiene la actual plataforma de apoyo a la docencia REKO, en las carreras de Diseño Industrial y Diseño en Comunicación Visual, tomando en cuenta su carácter emblemático en la Universidad Tecnológica Metropolitana, además del hecho, que no han logrado aplicar concretamente el sistema de aprendizaje virtual en todas sus asignaturas a pesar de que estas carreras, por su formación, requieren un conocimiento digital avanzado. Para ello se indagó en aspectos técnico gráficos y técnicos pedagógicos.

Sus resultados, respecto a lo técnico gráfico, se dividen en tres categorías: gráfica visual, usabilidad e interfaz y técnica digital web. En torno a la gráfica visual, se concluyó que la plataforma carece de una identidad propia institucional que permita ser fácilmente identificable por el usuario, ya sea profesor o alumno. Un sitio web de estas características debe ser asimilado por el usuario, por lo que debe asumir una cierta empatía que le permita familiarizarlo con otras plataformas y/o identificarlo fácilmente como una herramienta de apoyo y no de estorbo. En este punto, se entiende el rechazo de la plataforma por parte de los docentes. Ellos no logran entender cómo opera el sitio web, debido a una carencia gráfica y visual que les permita intuitivamente entender cómo se utiliza.

En el aspecto de usabilidad e interfaz, se destaca mucho la carencia de jerarquía y desorganización de los elementos. Al perder aspectos de estructura pierde también aspectos de orden, y por ello los recursos no son bien implementados, lo que provoca que los usuarios

El análisis de la plataforma virtual "REKO” de la Universidad Tecnológica Metropolitana del

Estado de Chile, desde la mirada de expertos y docentes.

Cristóbal Felipe Moreno Muñoz, Ximena Alejandra Díaz Orellana

Fabián Enrique Jeno Hernández

DOI: http://dx.doi.org/10.22458/caes.v8i1.xxxx

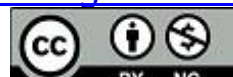

Artículo protegido por licencia Creative Commons 
REVISTA ELEETRÍNICA CALIDAD EN LA EDULACIÍN SULPERIIRR

Instituta de Gestión de la Calidad Académica

Universidad Estatal a Distancia, Casta Rica
ISSN: 1659-4703, VDL. 8(I) ENERD-JUNIT, 2017: 176 - 206

http://investiga.uned.ac.cr/revistas/index.php/revistacalidad

Correa: revistacalidad国uned. ac.сГ

DOI: http://dx.doi.org/10.22458/caes.v8i1.xxxx

del tipo docente, carentes del uso tecnológico, tiendan a confundir la información y pierdan el sentido didáctico de la plataforma.

Finalmente, en el aspecto de técnica digital web, según lo expuesto por los expertos, la plataforma cumple conformemente con los requerimientos que necesita una herramienta de estas características. Presenta versatilidad en cuanto a disponer de material didáctico de diversas tipologías, tamaños y contenidos; el sistema fluye bien y opera correctamente. Este factor es fundamental porque da a entender que esta sí tiene los recursos necesarios para operar de buena forma, lo que implica que el docente sabe y desea manipularla, y no debiese tener inconvenientes. Esto se contrapone directamente con lo que exponen los docentes en el grupo focal.

En torno al análisis pedagógico, se evaluaron elementos del actual entorno virtual de interacción educativa REKO, desde la mirada de académicos de las carreras de Diseño Industrial y Diseño en Comunicación Visual que deben utilizar este tipo de apoyo en la docencia. A partir de la opinión de los docentes participantes en el grupo focal surgieron las siguientes conclusiones:

El uso de la plataforma es en general solo como mensajería. Es poco efectiva, dado que este recurso no presenta las facilidades de acceso ni de usabilidad que los docentes consideran necesarias, ya que la mayoría de ellos no ha podido utilizarla como repositorio. Esta opinión se contrapone con aquella de los expertos, ya que, según el análisis de Recursos de

El análisis de la plataforma virtual “REKO” de la Universidad Tecnológica Metropolitana del

Estado de Chile, desde la mirada de expertos y docentes.

Cristóbal Felipe Moreno Muñoz, Ximena Alejandra Díaz Orellana

Fabián Enrique Jeno Hernández

DOI: http://dx.doi.org/10.22458/caes.v8i1.xxxx

(c) (i) $(5)$

Artículo protegido por licencia Creative Commons 
REVISTA ELECTRÍNICA CALIDAD EN LA EDULACIÍN SUPPERIIRR

Instituta de Gestión de la Calidad Académica

Universidad Estatal a Distancia, Casta Rica
ISSN: 1659-4703, VDL. 8(I) ENERD-JUNIT, 2017: 176 - 206

http://investiga.uned.ac.cr/revistas/index.php/revistacalidad

Correa: revistacalidad国uned. ac.сГ

DOI: http://dx.doi.org/10.22458/caes.v8i1.xxxx

Almacenamiento, (que se revisó en la tercera categoría del análisis técnico gráfico), estos son de fácil uso y poseen una variedad de recursos razonables.

Además, se encontró que la plataforma no se utiliza porque hay preferencia por lo presencial, según el modelo pedagógico tradicional. La totalidad de los docentes consultados consideran que la interacción y retroalimentación que se produce dentro del aula, es irremplazable por el uso de recursos tecnológicos. Sin embargo, el modelo pedagógico que se basa en el uso de recursos tecnológicos, propone que una plataforma virtual también posibilita la retroalimentación que existe entre docentes y estudiantes o entre los estudiantes mismos de manera efectiva.

Se debe considerar que la opinión de los docentes se contrapone con el interés que demuestran los alumnos actuales que, anteriormente, hemos llamado "nativos digitales". Estos últimos entienden que, el tipo de entrega de información tradicional, a través de clases magistrales y el uso de literatura en formato impreso, ya fue irremediablemente reemplazado por clases más interactivas y libros principalmente en formato digital. Por consecuencia, los recursos tecnológicos utilizados como apoyo al proceso enseñanza-aprendizaje, surgen como una fuerte herramienta que considera la diversidad de los individuos, sus intereses, valores intelectuales, habilidades y capacidades propias, al igual que su realidad particular. EI entorno virtual permite que cada participante elija su forma de participar y actuar, trabaje a su ritmo y consolide los conocimientos adquiridos. Le permite, además, al alumno adquirir progresivamente una autonomía en sus actividades habituales. Por lo tanto, el programa, sus

El análisis de la plataforma virtual “REKO” de la Universidad Tecnológica Metropolitana del

Estado de Chile, desde la mirada de expertos y docentes.

Cristóbal Felipe Moreno Muñoz, Ximena Alejandra Díaz Orellana

Fabián Enrique Jeno Hernández

DOI: http://dx.doi.org/10.22458/caes.v8i1.xxxx

$$
\text { (c) }(1)(8)
$$

Artículo protegido por licencia Creative Commons 
REVISTA ELECTRÍNICA CALIDAD EN LA EDULACIÍN SUPPERIIRR

Instituta de Gestión de la Calidad Académica

Universidad Estatal a Distancia, Casta Rica
ISSN: 1659-4703, VDL. 8(I) ENERD-JUNIT, 2017: 176 - 206

http://investiga.uned.ac.cr/revistas/index.php/revistacalidad

Carrea: revistacalidad国uned.ac.сГ

DOI: http://dx.doi.org/10.22458/caes.v8i1.xxxx

contenidos, las actividades, los proyectos, etc. deben adaptarse a las condiciones, necesidades y realidades cambiantes de los participantes y su entorno en comunidad educativa.

También se analizó la efectividad de la plataforma en cuanto al proceso de evaluación de la enseñanza-aprendizaje. Se concluyó que los aspectos del sistema de formación en línea favorecen autoevaluaciones con sistemas objetivos de calificación, que ayudarán al alumno a situarse en el nivel de aprendizaje conseguido y reconducir el proceso hacia niveles superiores de ejecución.

La evaluación del rendimiento del aprendizaje de los alumnos, en los sistemas basados en tecnología, es uno de los temas claves como en cualquier programa de formación. Cabe recordar que la mayor parte de los esfuerzos que se realizan sobre evaluación en línea del aprendizaje se centran en desarrollar herramientas informáticas como Quiz Test Creator, Web Work-Shett, WebTest, Question Mark Perception, etc., donde los planteamientos sobre evaluación están totalmente desligados del diseño general del programa de formación, salvo en algunos casos específicos ligados a sistemas de autor. En este sentido, podemos decir que las herramientas de evaluación están constituidas por softwares diseñados para la creación de pruebas tipo test, de respuesta cerrada; generalmente desarrolladas desde planteamientos tecnológicos, sin bases pedagógicas en su concepción. No consideran todo el proceso enseñanza-aprendizaje y, en caso de incluir un módulo de evaluación, este

El análisis de la plataforma virtual “REKO” de la Universidad Tecnológica Metropolitana del

Estado de Chile, desde la mirada de expertos y docentes.

Cristóbal Felipe Moreno Muñoz, Ximena Alejandra Díaz Orellana

Fabián Enrique Jeno Hernández

DOI: http://dx.doi.org/10.22458/caes.v8i1.xxxx

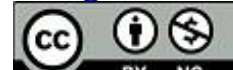

Artículo protegido por licencia Creative Commons 
REVISTA ELECTRÍNICA CALIDAD EN LA EDULACIÍN SUPPERIIRR

Instituto de Gestión de la Calidad Académica

Universidad Estatal a Distancia, Casta Rica
ISSN: 1659-4703, VQL. 8(I) ENERD-JUNID, 2017: 176 - 206

http://investiga.uned.ac.cr/revistas/index.php/revistacalidad

Carrea: revistacalidad国uned.ac.६Г

DOI: http://dx.doi.org/10.22458/caes.v8i1.xxxx

prácticamente se limita a los test como único instrumento para llevarlo a cabo. Además, carecen de base pedagógica y no se adaptan al alumno.

También debe considerarse que el proceso de evaluación ha de estar planificado, las estrategias de evaluación han de ser coherentes con los materiales aportados en línea y se han de presentar los criterios o referentes de evaluación de forma explícita. De esta manera, el alumno en línea puede informarse sobre lo qué se va a evaluar, ¿cómo? ¿cuándo? y bajo qué criterios será valorado su trabajo.

Por consecuencia, se requiere por parte de los alumnos un compromiso y una responsabilidad con respecto a su proceso de aprendizaje; y, por parte del tutor o coordinador, una motivación y vinculación con los contenidos. Es decir, debe estar presente virtualmente a través de mensajería instantánea, al igual que moderar y organizar los aportes de los participantes en línea.

Algunas reflexiones con respecto a las peculiaridades de la evaluación en ambientes virtuales tienen que ver con la interacción como base de estos entornos de aprendizaje, además, se debe de tomar en cuenta que, la acción docente en los modelos educativos que usan intensivamente las TIC's no es tarea de una persona, sino que es, esencialmente, tarea de la institución.

El análisis de la plataforma virtual "REKO” de la Universidad Tecnológica Metropolitana del

Estado de Chile, desde la mirada de expertos y docentes.

Cristóbal Felipe Moreno Muñoz, Ximena Alejandra Díaz Orellana

Fabián Enrique Jeno Hernández

DOI: http://dx.doi.org/10.22458/caes.v8i1.xxxx

(c) (i) (5)

Artículo protegido por licencia Creative Commons 
REVISTA ELECTRÍNICA CALIDAD EN LA EDULACIÍN SUPPERIIRR

Instituto de Gestión de la Calidad Académica

Universidad Estatal a Distancia, Casta Rica
ISSN: 1659-4703, VQL. 8(I) ENERD-JUNI0, 2017: 176 - 206

http://investiga.uned.ac.cr/revistas/index.php/revistacalidad

Carrea: revistacalidad国uned.ac.६Г

DOI: http://dx.doi.org/10.22458/caes.v8i1.xxxx

Al igual que en el proceso de enseñanza-aprendizaje se consideró que, en lo que respecta a las evaluaciones, los docentes consideran que la experiencia práctica es irremplazable, por las mismas razones mencionadas anteriormente. Sin embargo, como complemento y apoyo al proceso de enseñanza-aprendizaje, los recursos tecnológicos aún no están preparados para que permitan realizar todo tipo de actividades prácticas, entonces el recurso se podría utilizar como paso previo al desarrollo de actividades prácticas presenciales.

Se descubrió que la plataforma no está actualizada con respecto a otros recursos tecnológicos que disponen y utilizan los alumnos.

\section{Reflexiones y hallazgos}

Es importante considerar que, mediante las nuevas tecnologías, y de manera especial con Internet, se tiene acceso a información, pero no al conocimiento. Para analizar los efectos cognitivos y para promover efectos deseables debemos considerar, además de las potencialidades y limitaciones de cada medio, la propuesta educativa dentro de la cual está inmers; las actividades de aprendizaje propuestas y los contenidos a abordar. El aprendizaje ya no es el mismo cuando está soportado con las nuevas tecnologías. El diseño conceptual para introducir estas tecnologías al servicio de la educación es una tarea primordialmente pedagógico-comunicacional.

El análisis de la plataforma virtual “REKO” de la Universidad Tecnológica Metropolitana del

Estado de Chile, desde la mirada de expertos y docentes.

Cristóbal Felipe Moreno Muñoz, Ximena Alejandra Díaz Orellana

Fabián Enrique Jeno Hernández

DOI: http://dx.doi.org/10.22458/caes.v8i1.xxxx

(c) (i) (5)

Artículo protegido por licencia Creative Commons 
REVISTA ELEETRÍNICA CALIDAD EN LA EDULACIÍN SULPERIIRR

Instituta de Gestión de la Calidad Académica

Universidad Estatal a Distancia, Casta Rica
ISSN: 1659-4703, VDL. 8(I) ENERD-JUNIT, 2017: 176 - 206

http://investiga.uned.ac.cr/revistas/index.php/revistacalidad

Correa: revistacalidad国uned. ac.сГ

DOI: http://dx.doi.org/10.22458/caes.v8i1.xxxx

Ante esto, debemos diseñar y evaluar la introducción de nuevas tecnologías no solamente desde su aplicación educativa, sino también desde su función comunicativa. Debe mirarse el modelo de comunicación que subyace al sistema educativo específico, lo cual incluye la educación a distancia, la educación para los medios y la educación informal.

En todos estos sistemas sucede lo mismo: el aprendizaje se da en la medida en que el individuo se siente involucrado. Es por ello que el ambiente mediado por las tecnologías provoca procesos de aprendizaje. No es la tecnología misma sino el uso didáctico de ella, combinada con la práctica sobre medios, lo que promueve el aprendizaje.

El conectivismo presenta un modelo de aprendizaje que reconoce los profundos movimientos en una sociedad, en donde el aprendizaje ha dejado de ser una actividad interna e individual. Esta teoría de aprendizaje provee una mirada a las habilidades de aprendizaje y las tareas necesarias para que los alumnos desarrollen sus competencias en una era digital.

Esta investigación permitió también dar a conocer la estrecha relación que puede existir entre el mundo pedagógico y el mundo de la comunicación visual e informática, dando cuenta que la unidad de estos dos mundos puede ser fundamental para generar una conectividad más eficiente y eficaz entre el estudiante, el docente y los contenidos. Los aspectos visuales, perpetúales, propios del diseño gráfico y comunicacional, bien aplicados, pueden ser un importante aporte al uso de los contenidos pedagógicos virtuales, y probablemente un facilitador a la masificación de estos medios de aprendizaje.

El análisis de la plataforma virtual "REKO” de la Universidad Tecnológica Metropolitana del

Estado de Chile, desde la mirada de expertos y docentes.

Cristóbal Felipe Moreno Muñoz, Ximena Alejandra Díaz Orellana

Fabián Enrique Jeno Hernández

DOI: http://dx.doi.org/10.22458/caes.v8i1.xxxx

$$
\text { (c) }(1)(8)
$$

Artículo protegido por licencia Creative Commons 
REVISTA ELECTRÍNICA CALIDAD EN LA EDULACIÍN SUPPERIIRR

Instituta de Gestión de la Calidad Académica

Universidad Estatal a Distancia, Costa Rica
ISSN: 1659-4703, VQL. 8(I) ENERD-JUNID, 2017: 176 - 206

http://investiga.uned.ac.cr/revistas/index.php/revistacalidad

Correa: revistacalidad国uned. ac.сГ

DOI: http://dx.doi.org/10.22458/caes.v8i1.xxxx

La importancia de establecer ciertos criterios para un buen desarrollo de una plataforma virtual permite que esta investigación, pueda servir de base para tener en consideración los aspectos fundamentales que se deben tener presentes al momento de diseñarla y formularla.

Referencias

Altbach, P. Reisberg, Liz. \& Rumbley, L. (2009). Tras la pista de una revolución académica: Informe sobre las tendencias actuales para la Conferencia Mundial sobre la Educación Superior. Organizada por la UNESCO.

Bertoldi, S., Fiorito, M. \& Álvarez, M. (2006). Grupo focal y desarrollo local: aportes para una articulación teórico- metodológico. Ciencia Docencia y Tecnología, 17(33), 111-131. Recuperado de http:// www.revistacdyt.uner.edu.ar/pdfs/CDyT\%2033\%20\%20Pag\%20111-131\%20-\%20Grupo\%20Focal\%20y\%20Desarrollo\%20lo/ [Consultado 5 de septiembre de 2014].

Díaz, F. y Hernández, G. (2010). Estrategias Docentes para un aprendizaje significativo. (3 ed.) México D.F. México: Mc Graw Hill.

Díaz, X. y Moreno, C. (2014). La evaluación del actual entorno virtual de interacción educativa "REKO" de la Universidad Tecnológica Metropolitana en las carreras de Diseño Comunicación Visual y Diseño Industrial desde la perspectiva de distintos actores académicos. (Tesis de Postgrado). Santiago. Universidad Mayor, Facultad de Educación, Dirección de Postgrado.

Díaz, S. (2009): Plataformas educativas, un entorno para profesores y alumnos. Federación de Enseñanza de C.C.O.O. de Andalucía. Plataformas virtuales. Recuperado de http://www.youblisher.com/files/publications/7/37864/pdf.pdf/ [Consultado 4 de marzo de 2014].

El análisis de la plataforma virtual “REKO” de la Universidad Tecnológica Metropolitana del

Estado de Chile, desde la mirada de expertos y docentes.

Cristóbal Felipe Moreno Muñoz, Ximena Alejandra Díaz Orellana

Fabián Enrique Jeno Hernández

DOI: http://dx.doi.org/10.22458/caes.v8i1.xxxx

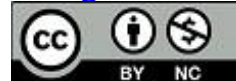

Artículo protegido por licencia Creative Commons 
REVISTA ELEETRÉNICA CALIDAD EN LA EDUCACIÍN SUPERIIRR

Instituta de Gestión de la Calidad Académica

Universidad Estatal a Distancia, Costa Rica
ISSN: 1659-4703, VDL. 8(I) ENERD-JUNIT, 2017: 176 - 206

http://investiga.uned.ac.cr/revistas/index.php/revistacalidad

Correa: revistacalidad国uned. ac.сГ

DOI: http://dx.doi.org/10.22458/caes.v8i1.xxxx

García, A. (2009). De la Teoría del Diálogo Mediado a la Práctica. X Congreso internacional sobre tecnología y educación a distancia. Tomo I. Editorial EUNED.

Gardner, Howard. (2005) Inteligencias múltiples: la teoría en la práctica. España: Paidos Ibérica.

Miles, M. B. y Huberman, A. M. (1984). Qualitative Data Analysis: A Sourcebook of New Methods. California: SAGE publications Inc.

Monsiváis, M. McAnally, L. \& Lavigne, G. (2014). Aplicación y validación de un modelo tecno pedagógico de formación docente mediante una plataforma educativa virtual. Revista de Universidad y Sociedad del Conocimiento (RUSC), 11(1). Universidad abierta de Cataluña. Barcelona.

Ramírez, I. (2011). Los Diferentes Paradigmas de Investigación y su Incidencia sobre los Diferentes Modelos de Investigación Didáctica, CONFERENCIA № 4. Diplomado internacional de didáctica y currículo. México.

Schank, R. (2013) Enseñando a pensar. Barcelona: Erasmus Ediciones..

Sepúlveda, N. y Calderón, I. (2007). Las TIC y los procesos de enseñanza-aprendizaje: la supremacía de las programaciones, los modelos de enseñanza y las calificaciones ante las demandas de la sociedad del conocimiento. Revista Iberoamericana de Educación n.ำ44/5. España.

Vaill, P. (1996). Aprendizaje como una manera de ser. San Francisco: Jossey - Blass Inc.

Vermunt, J. y Verloop, N. (1999). Congruence and friction between learning and teaching. Learning and Instruction. The Journal of the European Association for Research on Learning and Instruction (EARLI). Philadelphia, USA.5

World Economic Forum. Recuperado de https://www.weforum.org. [Consultado 5 de marzo de 2015].

El análisis de la plataforma virtual “REKO” de la Universidad Tecnológica Metropolitana del

Estado de Chile, desde la mirada de expertos y docentes.

Cristóbal Felipe Moreno Muñoz, Ximena Alejandra Díaz Orellana

Fabián Enrique Jeno Hernández

DOI: http://dx.doi.org/10.22458/caes.v8i1.xxxx

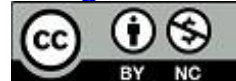

Artículo protegido por licencia Creative Commons 